\title{
Application of Spatio-Temporal Spectral Analysis for Detection of Seismic Waves in Gravitational-Wave Interferometer
}

\author{
Robert Szymko ${ }^{1}\left(\mathbb{D}\right.$, Mateusz Denys ${ }^{2, * \mathbb{D}}$, Tomasz Bulik ${ }^{3,4} \mathbb{D}^{\mathbb{D}}$, Bartosz Idźkowski ${ }^{4} \mathbb{D}_{\text {, Adam Kutynia }}$, $^{\text {, }}$ \\ Krzysztof Nikliborc ${ }^{4}$ and Maciej Suchiński ${ }^{4}$
}

check for

updates

Citation: Szymko, R.; Denys, M.; Bulik, T.; Idźkowski, B.; Kutynia, A.; Nikliborc, K.; Suchiński, M.

Application of Spatio-Temporal Spectral Analysis for Detection of Seismic Waves in Gravitational-Wave Interferometer. Galaxies 2021, 9, 50. https://doi.org/10.3390/galaxies 9030050

Academic Editor: Annalisa Allocca

Received: 9 April 2021

Accepted: 12 July 2021

Published: 14 July 2021

Publisher's Note: MDPI stays neutral with regard to jurisdictional claims in published maps and institutional affiliations.

Copyright: (C) 2021 by the authors. Licensee MDPI, Basel, Switzerland. This article is an open access article distributed under the terms and conditions of the Creative Commons Attribution (CC BY) license (https:/ / creativecommons.org/licenses/by/ $4.0 /)$.
1 Faculty of Physics, University of Warsaw, Pasteura 5, PL-02093 Warsaw, Poland; rszymko@gmail.com 2 Institute of Physics, Polish Academy of Sciences, Lotników Ave. 32/46, PL-02668 Warsaw, Poland

3 AstroCeNT-Particle Astrophysics Science and Technology Centre International Research Agenda, Nicolaus Copernicus Astronomical Center, Polish Academy of Sciences, Rektorska 4, PL-00614 Warsaw, Poland; bulik@camk.edu.pl

4 Astronomical Observatory, University of Warsaw, Ujazdowskie Ave. 4, PL-00478 Warsaw, Poland; bidzkowski@astrouw.edu.pl (B.I.); adam.kutynia@smartinstruments.pl (A.K.); nikli@wp.pl (K.N.); msuchinski@astrouw.edu.pl (M.S.)

* Correspondence: mateusz.denys@gmail.com

\begin{abstract}
Mixed spatio-temporal spectral analysis was applied for the detection of seismic waves passing through the west-end building of the Virgo interferometer. The method enables detection of a passing wave, including its frequency, length, direction, and amplitude. A thorough analysis aimed at improving sensitivity of the Virgo detector was made for the data gathered by 38 seismic sensors, in the two-week measurement period, from 24 January to 6 February 2018, and for frequency range 5-20 Hz. Two dominant seismic-wave frequencies were found: $5.5 \mathrm{~Hz}$ and $17.1 \mathrm{~Hz}$. The presented method can be applied for a better understanding of the interferometer seismic environment, and by identifying noise sources, help the noise-hunting and mitigation work that eventually leads to interferometer noise suppression.
\end{abstract}

Keywords: virgo; seismicity; seismic sensor; fourier analysis; newtonian noise; spatio-temporal spectral analysis

PACS: 04.80.Nn; 02.30.Nw; 91.30.Dk

\section{Introduction}

The first events detected by gravitational-wave (GW) detectors in the past decade have given us hope to answer the most fundamental questions of astronomy and physics, concerning, e.g., evolution of galaxies and black holes, gravitational collapse of supernovae, and limits of general relativity [1-6]. However, noise reduction in these detectors is a most complex task, due to very low amplitude of the incoming signals. Seismic characterization of the vicinity of the GW detectors is necessary for the noise reduction. The method presented in this paper enables the identification of such signals. This characterization can help noise mitigation, especially for Newtonian noise coming from fluctuating gravitational forces caused by density perturbations of the ground and of the atmosphere [7-10]. The data for this paper are seismic data of Newtonian noise array of the seismic sensors installed at west-end building (WEB) of the Virgo GW detector [11]. Virgo arms named West and North are orientated approx. $20^{\circ}$ clockwise from North. The Virgo coordinate system is centered on the beam splitter in the central building.

Spatio-temporal spectral analysis is a straightforward extension of harmonic analysis to more than one dimension. Apart from a temporal dimension, additional spatial dimensions are introduced. The method allows identification of the frequencies and the wave vectors of waves passing through the Virgo detector. The input data for our analysis contains spatial and temporal dimensions. Spatial part should have fixed boundaries. In such 
cases we can look for modes of variability, i.e., underlying spatio-temporal structures with preferred spatial pattern and temporal variation. If the signal is harmonic (wavelike), then we expect spatio-temporal spectral analysis to isolate any such modes that are present in the data. For instance, if we make spatio-temporal spectral analysis of a string instrument, we expect to find a definite relationship between the length scales and the time scales of the oscillations [12].

The method presented in this article is based on paper [13] and refs. therein, where seismic-noise structure has been estimated using arrays of detectors (see also [14]). Spatiotemporal spectral analysis has been used before to obtain the Rayleigh wave phase velocity dispersion curves [15]. It is also a widely explored method in the atmospheric sciences [16-20]. Moreover, [21] applied spatio-temporal spectral analysis to modeling rainfall fields. As far as only spatial Fourier transform is concerned, Kim and Barros [22] used its two-dimensional version to analyze the structure of soil moisture fields in different time moments, considered separately.

Investigations of the seismic characteristics of the Virgo site have been made previously in [23-25]. For recent works about seismic-noise measurements for gravitational-wave detectors see, e.g., [26-29].

This paper is structured as follows. The system of seismometers that we used for data acquisition is presented in Section 2. Then, we provide information about data analysis process in Section 3. The results of this analysis are described in Section 4. Finally, concluding remarks are given in Section 5 .

\section{Measuring System}

For this study, a system of seismic sensors in the west-end building (WEB) of the Virgo interferometer was examined. The system consists of 38 Innoseis Tremornet seismometers [30], deployed on the building floor. The exact coordinates of the seismometers are provided in Appendix A. The average distance between the seismometers was approximately $9.5 \mathrm{~m}$. Point $(0,0)$ of the coordinate system is the interferometer beam splitter mirror. The axes coincide with the Virgo arms.

The floor is supported by $30 \mathrm{~m}$ long poles, while the central platform and the basement are supported by a set of longer poles reaching a more stable gravel layer, at $52 \mathrm{~m}$ depth. Seismometers no. 1-23 were placed on the floor of the building, no. 24-38-on the central platform, while no. 37 and 38-in the basement. Vacuum chamber rests on the central platform at WEB (and north-end building as well) where one of the four mirror test masses of Virgo is located and suspended to a vibration isolation system. The construction also provides suppression of seismic noise above $15 \mathrm{~Hz}$ on the central platform. For frequency values above ca. $15 \mathrm{~Hz}$, there is a significant difference in the amplitude spectral densities (ASDs) between the platform and the floor [29].

The sensors were placed on the floor fixing their mount plate with double-sided adhesive tape for a good connection to ground. The sensors are $4.5 \mathrm{~Hz}$ geophones and have a flat response function in the range from 4.5 to at least $50 \mathrm{~Hz}$ where we present this analysis. Each sensor measure, the vertical ground velocity [30] and also contains a preamplifier and a 24-bit analog to digital converter. The digital signal of each sensor is transferred to a centralized unit through ethernet cable and then stored. The seismometers measure voltage changes in the range between $-5 \mathrm{~V}$ and $5 \mathrm{~V}$ with sensitivity (manufacturer defined) $77.3 \mathrm{~V} / \mathrm{m} / \mathrm{s}$. Seismometer signals were converted from volts to $\mathrm{m} / \mathrm{s}$, by using the appropriate conversion coefficient $5 /\left(2^{23} \times 77.3\right)$. For more details see [31].

The data that were used for the calculations were collected during a two-week measurement period, from 24 January to 6 February 2018. The data of each sensor are recorded with $500 \mathrm{~Hz}$ sampling frequency. Not all the 256 1-h data files generated in the measurement period are complete. The effective measurement time is $250 \mathrm{~h}$ ( $\sim 10.5$ days). A series of 10 consecutive zeros was assumed being empty-data period for this sensor. Empty periods amount to $3.4 \%$ of the total data. 


\section{Methodology}

We indicate with $\psi(x, y, t)$ the vertical velocity measured at time $t$ of the seismometer positioned at coordinates $(x, y)$. We apply the mixed spatio-temporal Fourier transform (FT) to obtain the quantity $\mathrm{FT}\left(k_{x}, k_{y}, \omega\right)$, where $k_{x}, k_{y}$ are the components of the wavevector $\overrightarrow{\mathbf{k}}=\frac{2 \pi}{\lambda} \hat{\mathbf{r}}$, where $\lambda$ is the wavelength, and $\omega$ is the wave frequency. The spatiotemporal transform is defined as:

$$
\mathrm{FT}\left(k_{x}, k_{y}, \omega\right)=\int d t \int d x \int d y \psi(x, y, t) e^{-i\left(\omega t+k_{x} x+k_{y} y\right)}
$$

Time $t$ is discretized with $\Delta t=0.002 \mathrm{~s}$. The value of $\mathrm{FT}\left(k_{x}, k_{y}, \omega\right)$ provides information about the amplitude of the particular wave.

In general, the spatio-temporal Fourier transform consists of wave vectors for each wave detected in the signal (represented along the $k_{x}, k_{y}$ axes) together with the frequency of that wave (on $\omega$ axis). Therefore, we can obtain length, direction, frequency and amplitude (The wave amplitude can be obtained by calculating an inverse Fourier transform of each ASD peak in the final result. However, we must consider in this case all rescalings done during the process of the FT calculation-see below in this section.) of a wave that passed through the measuring system during the considered time period. Notably, Rayleigh surface waves are expected to prevail in the seismic wavefield in the vicinity of the Virgo west-end building [32]. Therefore, we can neglect vertical differences between the spatial positions of the seismometers ( $z$ axis).

Remarkably, spatio-temporal Fourier transform, as any other multi-dimensional Fourier transform, can be computed in two steps. Having the input data of $(x, y, t)$-points, we can calculate, first, only the temporal part [obtaining $(x, y, \omega)$-points] and subsequently we can calculate the spatial part, obtaining as final result the $\left(k_{x}, k_{y}, \omega\right)$-points.

In the approach presented herein, at first, the average value of the signal was subtracted from the input, for each sensor separately. Then, the whole signal was divided into 20-s long segments (time frames), 10,000 samples each. By dividing the data into short pieces, we can detect short wave impulses, lasting only a couple of seconds. Due to the fact that the spatio-temporal method, as any other Fourier-based method, uses finite sets of data, the questions of spectral leakage and, consequently, the necessity of applying appropriate windowing functions should be considered to avoid artificial components to appear in the final result. For that purpose the Hann window function was used.

After the initial preparation of data, explained above, the time-frequency part, for every time frame and for each sensor separately, was calculated, using FFT (Fast Fourier Transform) algorithm. After that, the result was multiplied by the appropriate sensor response function, which depends on the frequency value $\omega$. Subsequently, the spatial part was calculated, variables $(x, y)$ were changed to $u=s_{f} x$ and $v=s_{f} y$, where

$$
s_{f}=\frac{\pi}{\max \left\{|x|_{\max },|y|_{\max }\right\}},
$$

so that both spatial dimensions were normalized to $(-\pi, \pi)$ range. $((-\pi, \pi)$ range is needed for NUFFT algorithm—see Section 4).

Finally, the variables were changed back to $k_{x}=s_{f} k_{u}, k_{y}=s_{f} k_{v}$, and three-dimensional complex modulus of the spectrum was calculated to obtain the 3D amplitude spectral density (ASD):

$$
\operatorname{ASD}\left(k_{x}, k_{y}, \omega\right)=\left|\mathrm{FT}\left(k_{x}, k_{y}, \omega\right)\right|(T X Y)^{-1 / 2},
$$

where $\operatorname{FT}\left(k_{x}, k_{y}, \omega\right)$ is the spatio-temporal Fourier transform of the measurement data defined in Equation (1), and $T, X, Y$ are the temporal and spatial extent of the data respectively. 


\subsection{Test Data}

To test the algorithm, the following simulation was run. We created a simulated set of data adopting the same $(x, y)$ positions of the seismometers the superposition of two sinusoidal waves:

$$
\psi(x, y, t)=A \cos \left(k_{x} x-\omega_{1} t\right)+B \cos \left(k_{y} y-\omega_{2} t\right),
$$

where $k_{x}=0.578 \mathrm{~m}^{-1}, k_{y}=-0.876 \mathrm{~m}^{-1}, \omega_{1}=3(2 \pi) \mathrm{Hz}, \omega_{2}=5(2 \pi) \mathrm{Hz}$, and for instance $A=B=1000 \mathrm{~V}$. (To mimic the seismometer measurements in volts, approximated to integer values, due to the fact that the real seismometer measurements in volts were integer values. However, before the process of ASD calculation, the units were converted from volts to $\mathrm{m} / \mathrm{s}-c f$. Section 3.) The artificial data in volts are converted to $\mathrm{m} / \mathrm{s}$.

Figure 1 shows the obtained spatio-temporal amplitude spectral density for these exemplary data, in a form of colormap, for 3, 4, and $5 \mathrm{~Hz}$. Additionally, a rank of ASD values is shown for frequency $3 \mathrm{~Hz}$. The right panel of Figure 1a shows the inverse cumulative plot of all the points from the left panel, ordered from the largest to the smallest ASD value. The red dot represents the maximum corresponding to the yellow point in the left panel. This illustrates that the numerical noise due to non-uniform spatial sensor grid is negligible.
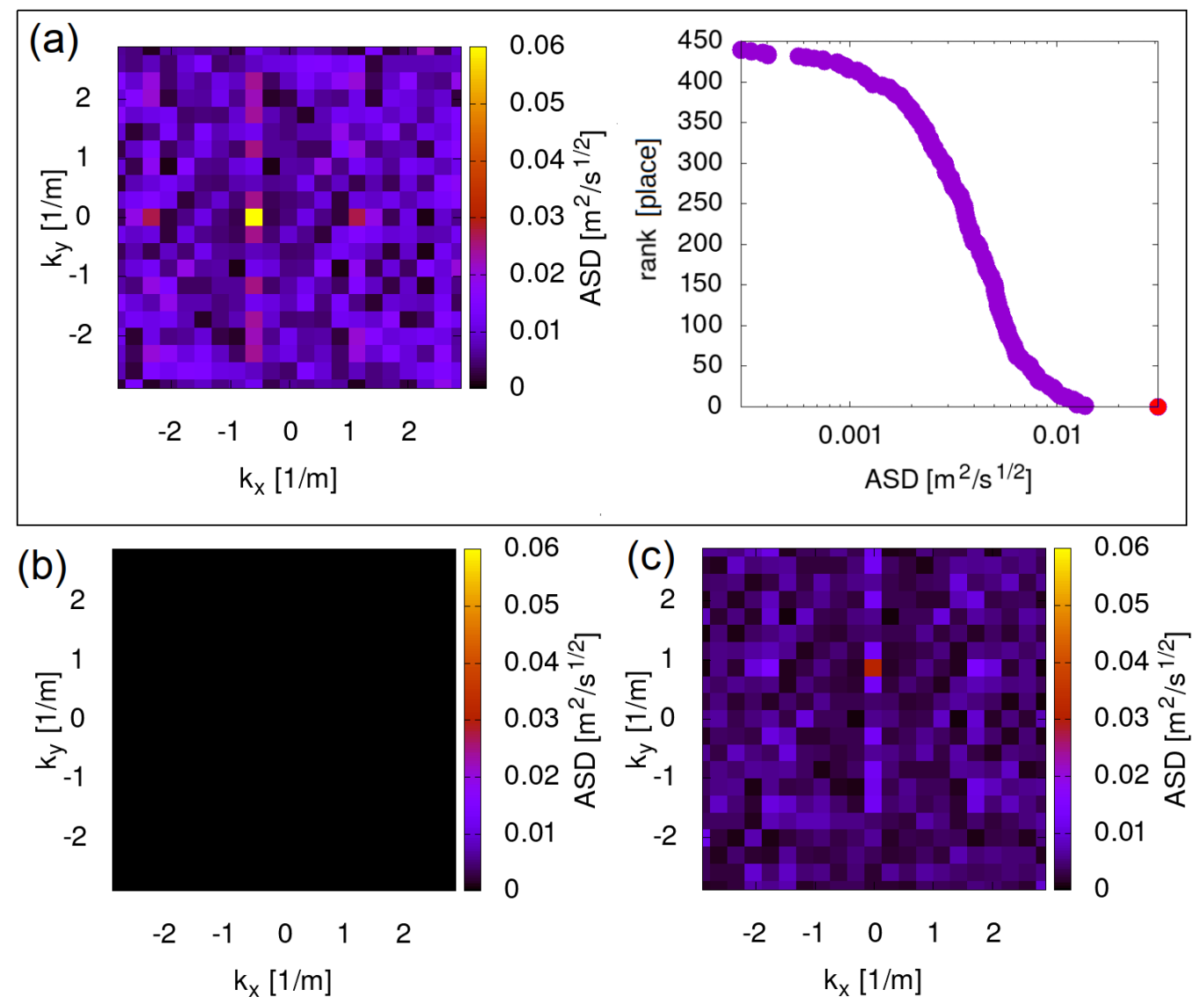

Figure 1. Colormap of amplitude spectral density (arbitrary units) for a superposition of two sinusoidal waves given by Equation (4) (where $k_{x}=0.578 \mathrm{~m}^{-1}, k_{y}=-0.876 \mathrm{~m}^{-1}, \omega_{1}=3(2 \pi) \mathrm{Hz}$, $\omega_{2}=5(2 \pi) \mathrm{Hz}, A=B=1000 \mathrm{~V}$ ) for (a) $3 \mathrm{~Hz}$ (on the left; on the right, additionally, a rank of ASD values in semi-log scale is shown, where the outstanding point corresponding to the yellow peak on the left is marked in red), (b) $4 \mathrm{~Hz}$, (c) $5 \mathrm{~Hz}$.

As expected, the spatio-temporal amplitude spectral density for such strong signal consists of two outstanding peaks for frequency $3 \mathrm{~Hz}$ and $5 \mathrm{~Hz}$ (Figure $1 \mathrm{a}, \mathrm{c}$ ), corresponding 
to both parts of the superpositioned wave, with proper wave-vector and frequency values. Both peaks are outstanding compared to the other spatial modes for frequencies $3 \mathrm{~Hz}$ and $5 \mathrm{~Hz}$. The peaks that correspond to $3 \mathrm{~Hz}$ and $5 \mathrm{~Hz}$ are larger than the average value by about 10 times the standard deviation of the data. We also present the rank plot for the case of $3 \mathrm{~Hz}$, Figure 1a on the right, the outstanding $\mathbf{k}$-value is denoted by the red dot in the bottom right corner, which stands above the remaining data significantly. As expected, no outstanding mode is found at $4 \mathrm{~Hz}$ [Figure $1 \mathrm{~b}$ ]. We conclude that the method is robust with respect to identification of the frequency of the waves, but it introduces some noise related to the non-uniform spatial grid.

\subsection{Measurement Data}

After the successful test of the algorithm on the artificial data, the analysis of the real data collected from the Virgo west-end building was made. In the initial step, the seismometer indications in volts were converted to the appropriate values in $\mathrm{m} / \mathrm{s}$ (cf. Section 3 ). Then, the three-dimensional transforms for these data were calculated using FFT and NUFFT algorithms (cf. Section 3). The Non-Uniform FFT algorithm (NUFFT) [33-35] is the version of FFT algorithm for non-uniform samples, as the locations of the seismometers were not uniform. A version of the NUFFT algorithm for two dimensions was applied. Finally, the spatio-temporal amplitude spectral density was calculated for each $20-\mathrm{s}$ period and each frequency. An example plot is usually characterized by one main maximum-cf. Figure 2 .

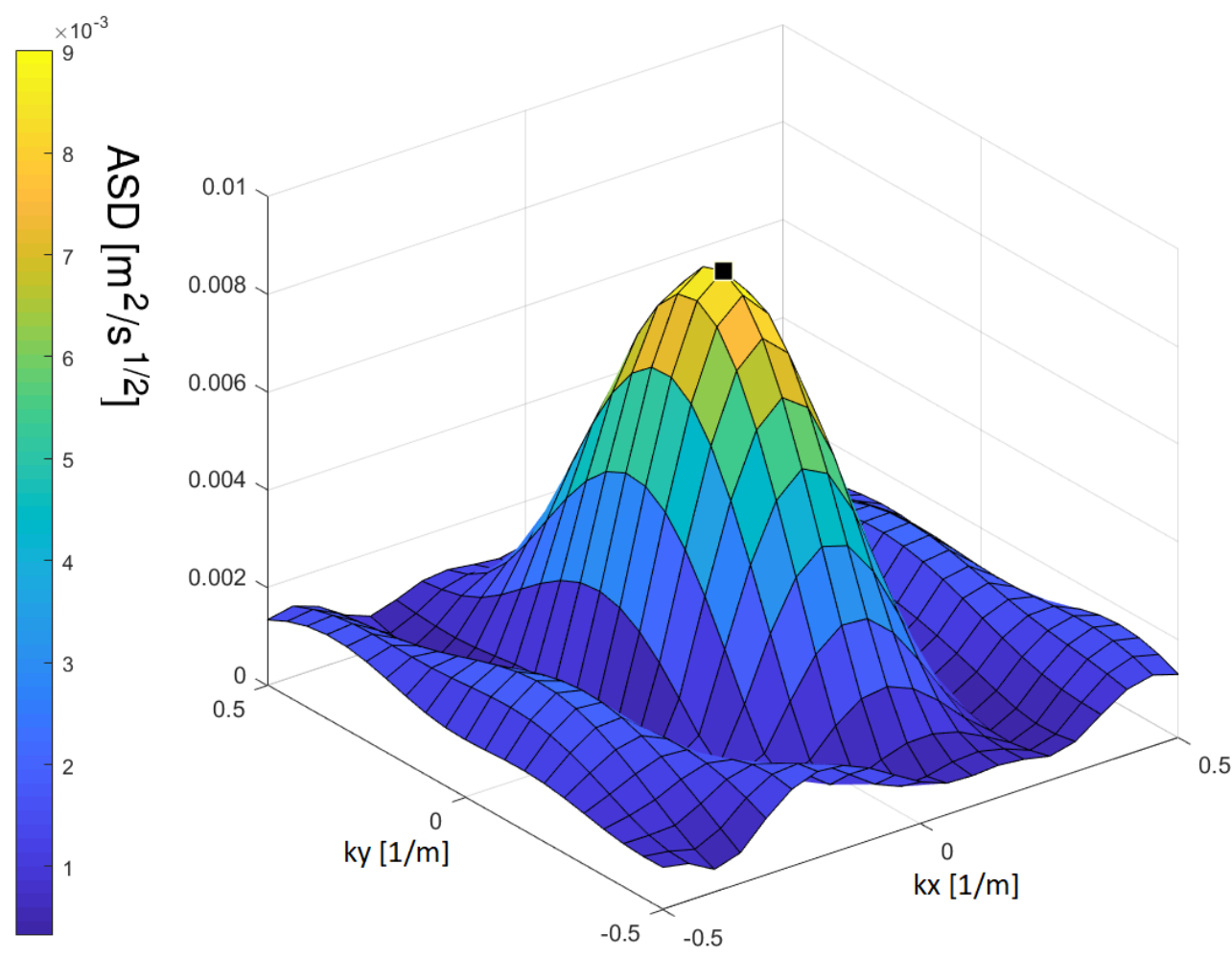

Figure 2. ASD value in $\left(k_{x}, k_{y}\right)$ space for frequency $5.5 \mathrm{~Hz}, 10$ th min of the first hour of measurements (marked also using the color scale on the left).

The next step was finding a position $\left(k_{x}, k_{y}\right)$ of maximal ASD value in time, for each frequency from $5 \mathrm{~Hz}$ to $20 \mathrm{~Hz}$, step $0.1 \mathrm{~Hz}$. For the data from a dozen hours of the experiment, a wide range of the wave-vector $\mathbf{k}$ was checked. Over $95 \%$ of the maximum ASD values found for any frequency in each 20-s period are in the range $\mathbf{k}$ from -0.5 to $0.51 / \mathrm{m}$ (Figure 3). Moreover, the maxima found outside this inner range were very low and likely corresponded to noise. Therefore, we have adopted this smaller range for all calculations. 


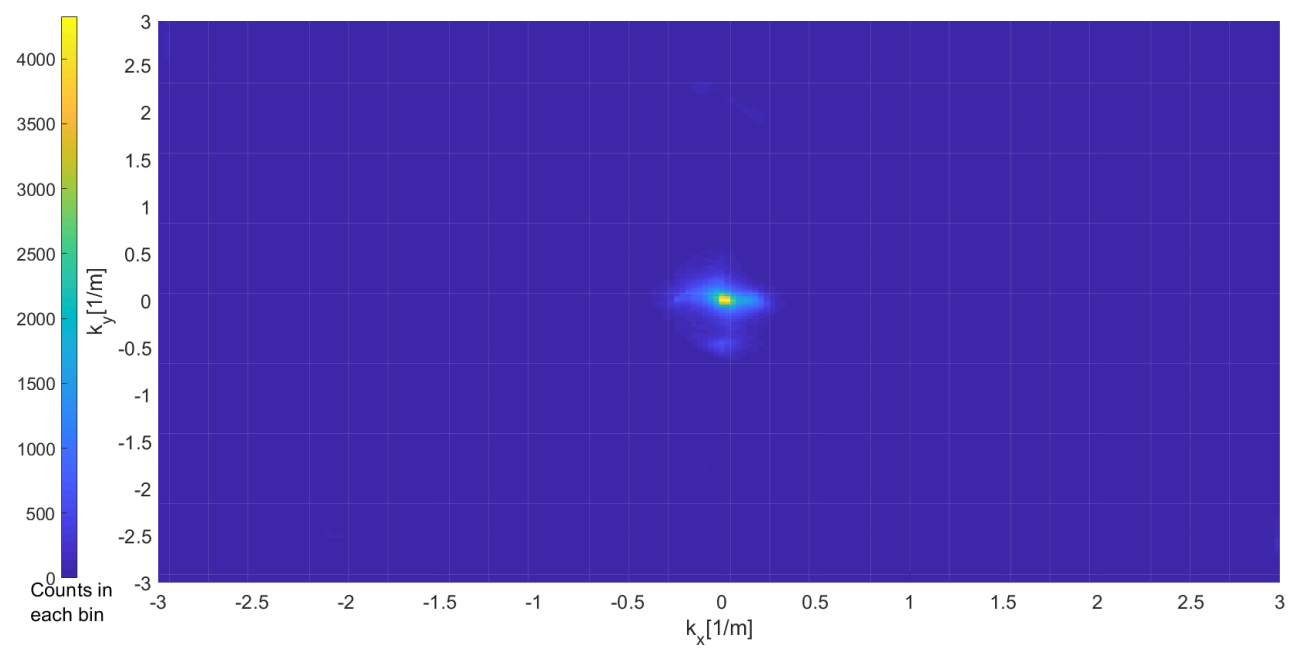

Figure 3. Positions of the maximum ASD of any frequency for data from a dozen hours of the experiment. Most of points are in center of plot in the range from -0.5 to $0.51 / \mathrm{m}$.

Examples of preliminary results for 20-s pieces of seismic data, taken from the same hour of measurement, are shown in Figure 4.
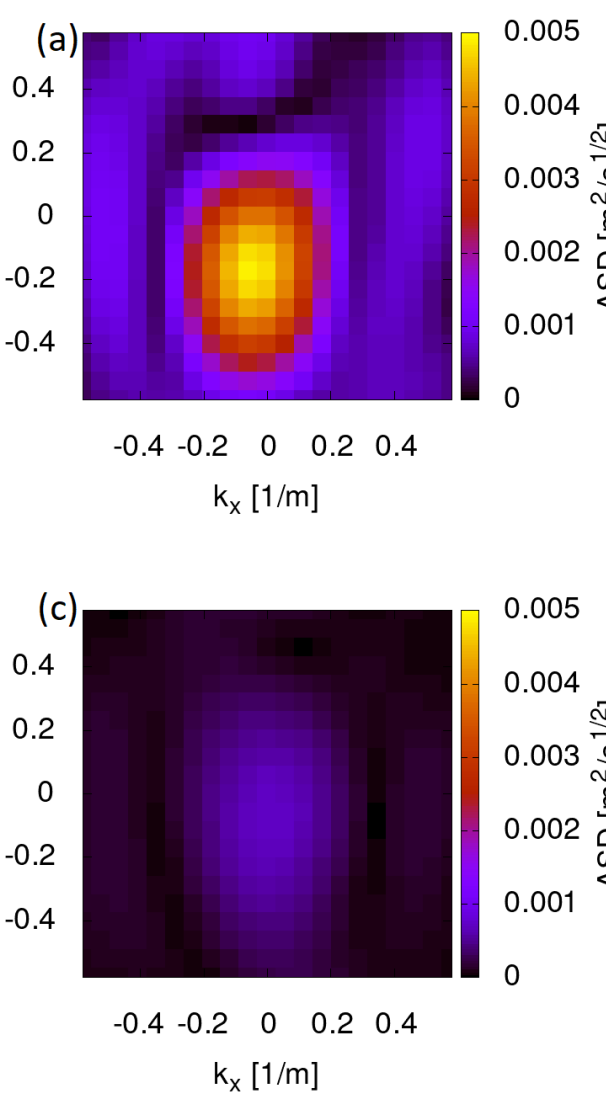
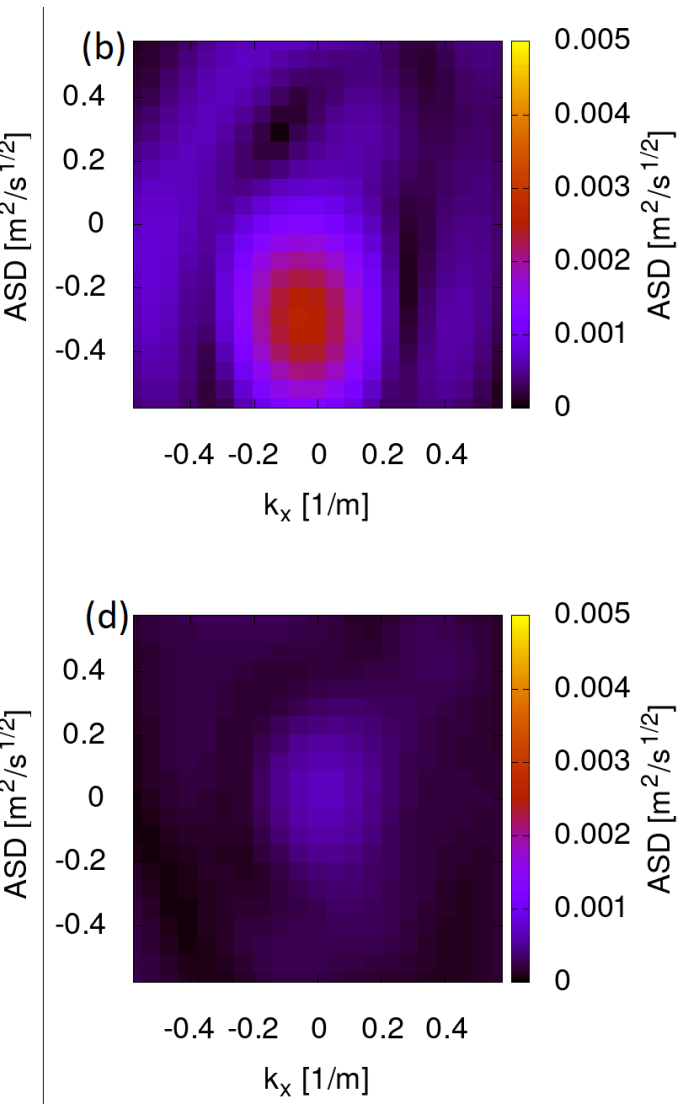

Figure 4. Colormap of ASD in $\left(k_{x}, k_{y}\right)$ space for 20-s pieces of seismic data from 24 January 2018, 13:30, and for: (a) $5 \mathrm{~Hz}$, (b) $10 \mathrm{~Hz}$, (c) $12 \mathrm{~Hz}$, (d) $15 \mathrm{~Hz}$.

There are dominant waves for particular frequencies, as expected. In the first plot for $5 \mathrm{~Hz}$ [Figure 4a] there is a strong maximum. In Figure $4 \mathrm{~b}$ for $10 \mathrm{~Hz}$ there is much weaker and fuzzy maximum. For Figure $4 \mathrm{c}, \mathrm{d}(12 \mathrm{~Hz}$ and $15 \mathrm{~Hz}$, respectively) there are not any signals visible, but rather some artifacts present due to inaccuracy of the measurement or the NUFFT method used. 


\subsection{Dominant Waves}

Resolution of wave-vector space and frequencies taken for our calculation is $\delta_{k}=0.05 \mathrm{~m}^{-1}$, $\delta_{\omega}=0.1 \mathrm{~Hz}$, and we investigated the region of wave-vector space with the size of $-0.5 \mathrm{~m}^{-1}<k_{x}, k_{y}<0.5 \mathrm{~m}^{-1}$, thus, for each frequency, there were 180 matrices $21 \times 21$ were obtained per hour of data. In order to determine dominant waves the following function (paraboloid, usually looks like our results from Figure 2) was fitted to the spatiotemporal Fourier spectra:

$$
\mathrm{f}(u, v)=A-{\frac{u^{2}}{\sigma_{u}}-v^{2}}_{\sigma_{v}},
$$

where $\mathrm{A}$ is maximal amplitude for a given matrix $21 \times 21, \sigma_{u}$ and $\sigma_{v}$ are proportional to standard deviations of $u$ and $v$, respectively,

$$
\begin{aligned}
& u=\left(k_{x}-k_{x 0}\right) \times \cos (\alpha)-\left(k_{y}-k_{y 0}\right) \times \sin (\alpha), \\
& v=\left(k_{x}-k_{x 0}\right) \times \sin (\alpha)+\left(k_{y}-k_{y 0}\right) \times \cos (\alpha),
\end{aligned}
$$

$k_{x 0}, k_{y 0}$ are coordinates of maximal ASD value, $\alpha$ is inclination of the paraboloid axis with respect to the $X$ axis, and $k_{x}, k_{y}$ are the matrix coordinates.

To increase the resolution in wave-vector space, for each matrix an area of $100 \times 100$ points close to the maximum was inspected, with an accuracy of localization of $\max \left\{\operatorname{ASD}\left(k_{x}, k_{y}\right)\right\}$ increased by two orders of magnitude. Then, the likelihood function:

$$
L=\sum_{i, j=1}^{21}\left[\mathrm{f}\left(k_{x}, k_{y}, k_{x 0}, k_{y 0}, \sigma_{u}, \sigma_{v}, \alpha, A\right)-\lambda(i, j) / \sigma_{L}\right]^{2},
$$

was calculated, where $\lambda(i, j)=\operatorname{ASD}\left(k_{x}, k_{y}, \omega\right)$ is the value of ASD-matrix element for a particular $\omega$, and $\sigma_{L}=0.05 \times A$ is assumed uncertainty of the experiment. (A change of $\sigma_{L}$ does not change coordinates of the ASD maximum, just its value.) The values of $\sigma_{u}$ and $\sigma_{v}$ in Equation (5) were tested in the range 50 to $150 \mathrm{~s}^{1 / 2} \mathrm{~m}^{-4}$. After normalization, collected values of $L$ were aggregated in the following way (Right-hand side of Equation (8) does not include $k_{x}, k_{y}$ as they reflect indices of summation $i, j$ in Equation (7), and $A$, as it was constant for all calculated $L$ values.)

$$
\begin{aligned}
L_{x, y}\left(k_{x 0}, k_{y 0}\right) & =\sum_{\sigma_{u}, \sigma_{v}, \alpha} L\left(k_{x 0}, k_{y 0}, \sigma_{u}, \sigma_{v}, \alpha\right), \\
L_{x}\left(k_{x 0}\right) & =\sum_{k_{x 0}} L\left(k_{x 0}, k_{y 0}\right), \\
L_{y}\left(k_{y 0}\right) & =\sum_{k_{y 0}} L\left(k_{x 0}, k_{y 0}\right) .
\end{aligned}
$$

Finally, for each frequency and each 20-s period of the measurement the cumulative distribution function was compute for $L_{x} i L_{y}$ which results in the coordinates $\left(k_{x}, k_{y}\right)$ of ASD maximum.

\section{Results}

In this section, the final results of the analysis of all data gathered between 24 January 2018 and 6 February 2018 are presented. Figure 5 shows dominant frequencies for the whole period. We have decided not to use the data below $5 \mathrm{~Hz}$ since the sensor resonant frequency is $4.5 \mathrm{~Hz}$ and their response below is decreasing. 


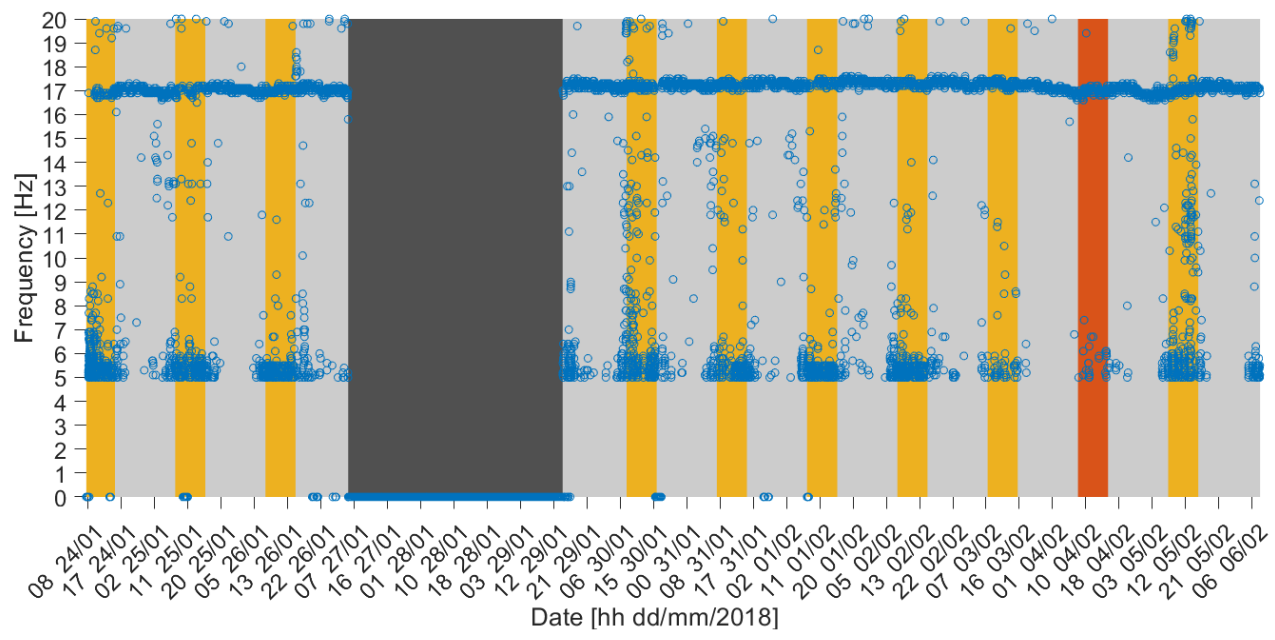

Figure 5. Frequency of ASD maximum for each time moment. The hours between 8 a.m. and 4 p.m. each day are indicated with yellow color and light grey represent the night hours, Sundays are indicated with orange, dark grey is a period of measurements break.

In Figure 6 a spectral histogram of the data is shown, i.e., a two-dimensional distribution of $\max (\mathrm{ASD})$ values vs. dominant frequencies. Apparently, there are two main frequencies at which ASD maxima accumulate: ca. $5-6 \mathrm{~Hz}$ and ca. $17 \mathrm{~Hz}$. The first one is characterized by strong day-night periodicity. As for the $5.5 \mathrm{~Hz}$ wave, its peak in frequency domain is very sharp, while for $17.1 \mathrm{~Hz}$ it is more blurred.

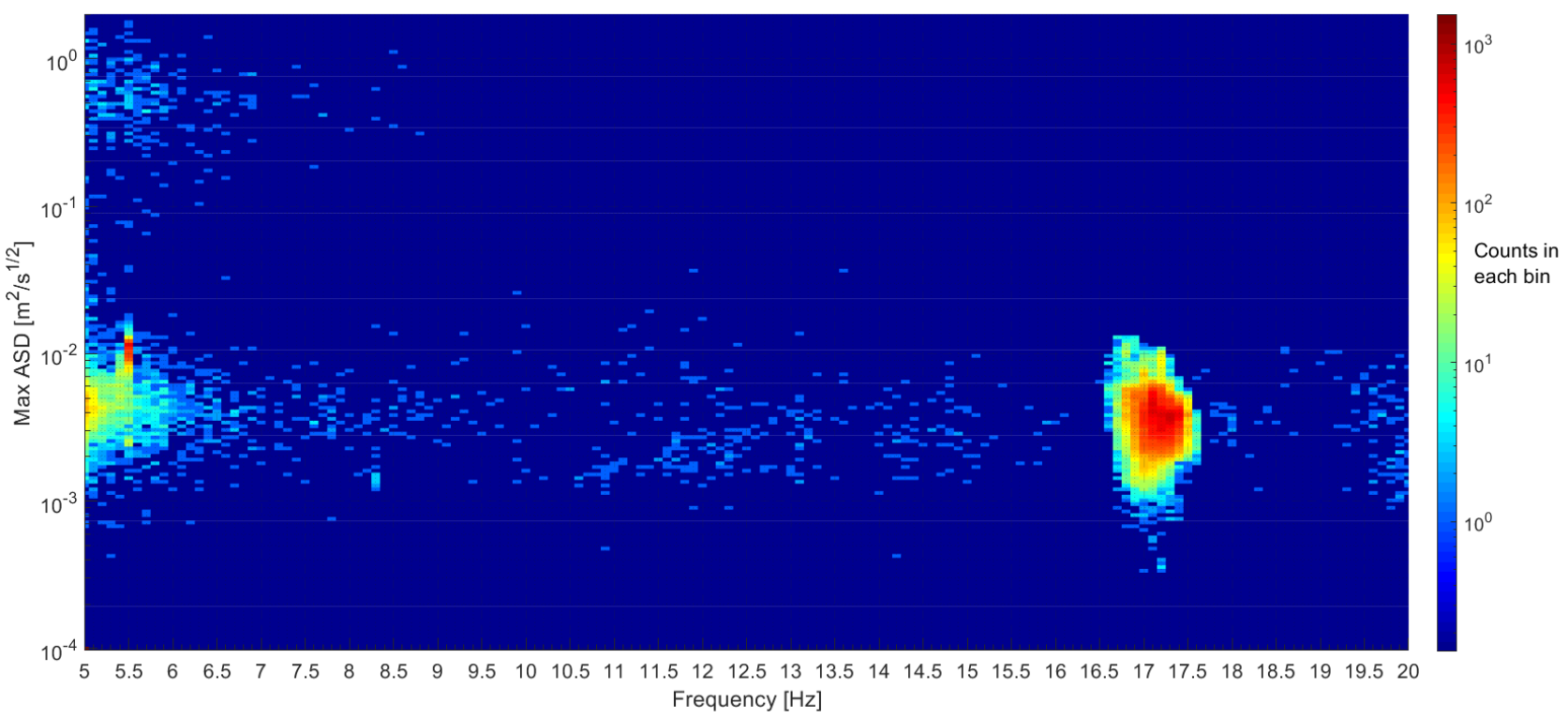

Figure 6. Spectral histogram of maximal ASD values vs. dominant frequencies. Bin width for dominant frequencies (horizontal axis) is 0.1 , for $\max (\mathrm{ASD})$ (vertical axis) it changes logarithmically from 0.0001 to 10 (200 bins in total).

Using methodology described in Section 3.3 coordinates of each max(ASD) in the wave-vector space, for particular frequencies and 20-s periods of the measurement, were found. Results are shown in Figure $7 \mathrm{a}-\mathrm{c}$ for $17.1 \mathrm{~Hz}$ and Figure $7 \mathrm{~d}-\mathrm{f}$ for $5.5 \mathrm{~Hz}$. 

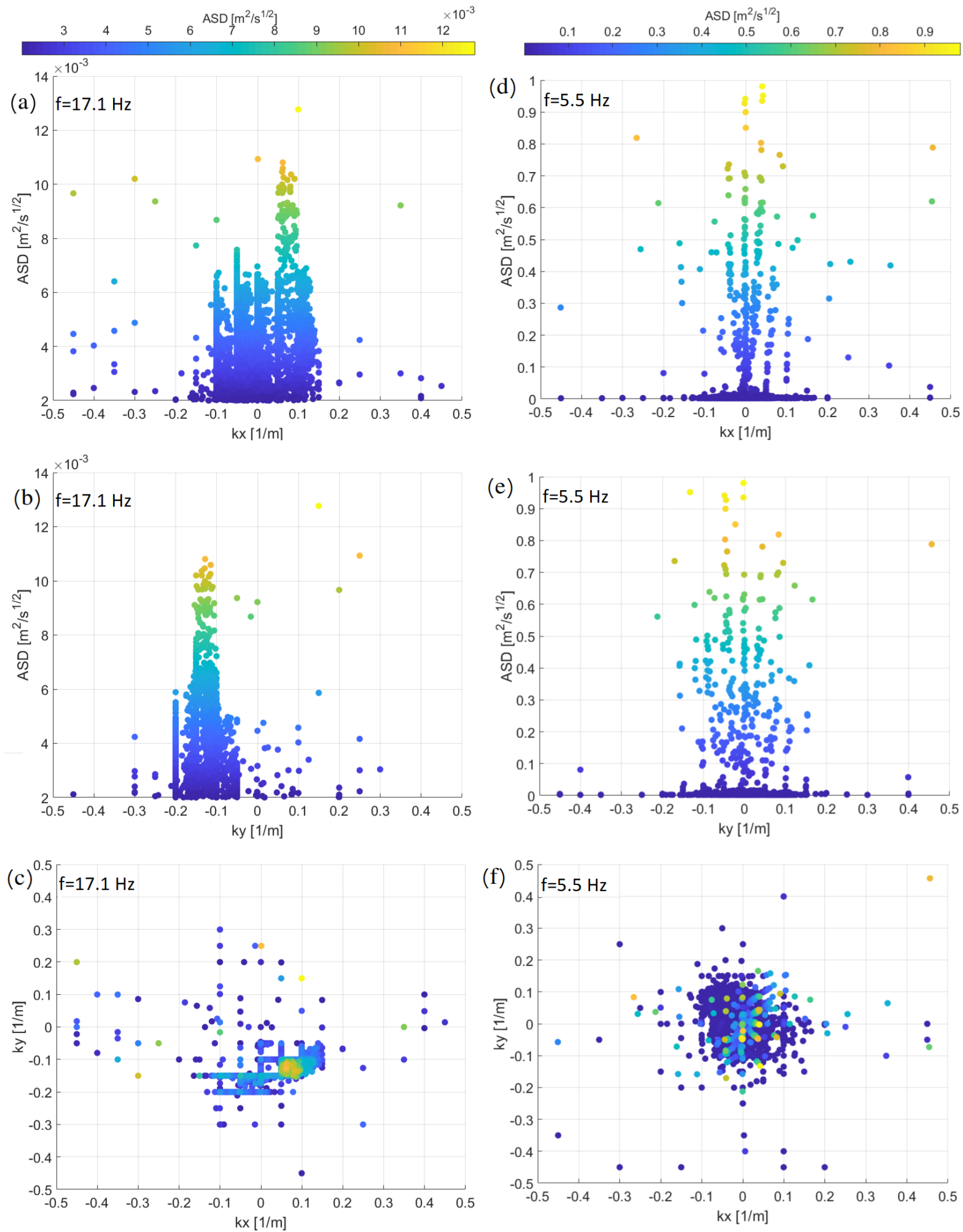

Figure 7. Locations of $\max (\mathrm{ASD})$ in wave-vector space for frequency $17.1 \mathrm{~Hz}$ projected onto (a) $X Z$, (b) $Y Z$, (c) $X Y$ surface and for frequency $5.5 \mathrm{~Hz}$ projected onto (d) $X Z,(\mathbf{e}) Y Z,(\mathbf{f}) X Y$ surface where $X$ and $Y$ reflect $k_{x}$ and $k_{y}$, respectively, and $Z$ reflects ASD. Colors indicate ASD values.

For the sake of clarity, the results are projected on $X Z, Y Z$, and $X Y$ surfaces, where $X$ and $Y$ correspond to $k_{x}$ and $k_{y}$, respectively, and $Z$ reflects ASD. To account for measurement and computation uncertainty we set a lower limit for $\max (\mathrm{ASD})$ to $0.5 \%$ of the maximal ASD value for all data, which was equal to $2.13 \mathrm{~m}^{2} / \mathrm{s}^{1 / 2}$. 
Figures 8 and 9 for frequencies $17.1 \mathrm{~Hz}$ and $5.5 \mathrm{~Hz}$, respectively, are histograms of wave direction and wavenumber. $0^{\circ}$ on upper plots does not indicate cardinal direction, but direction of $X$ axis in the seismometer coordination system presented in Table A1. This direction is also marked in Figure 10 in comparison with cardinal direction.
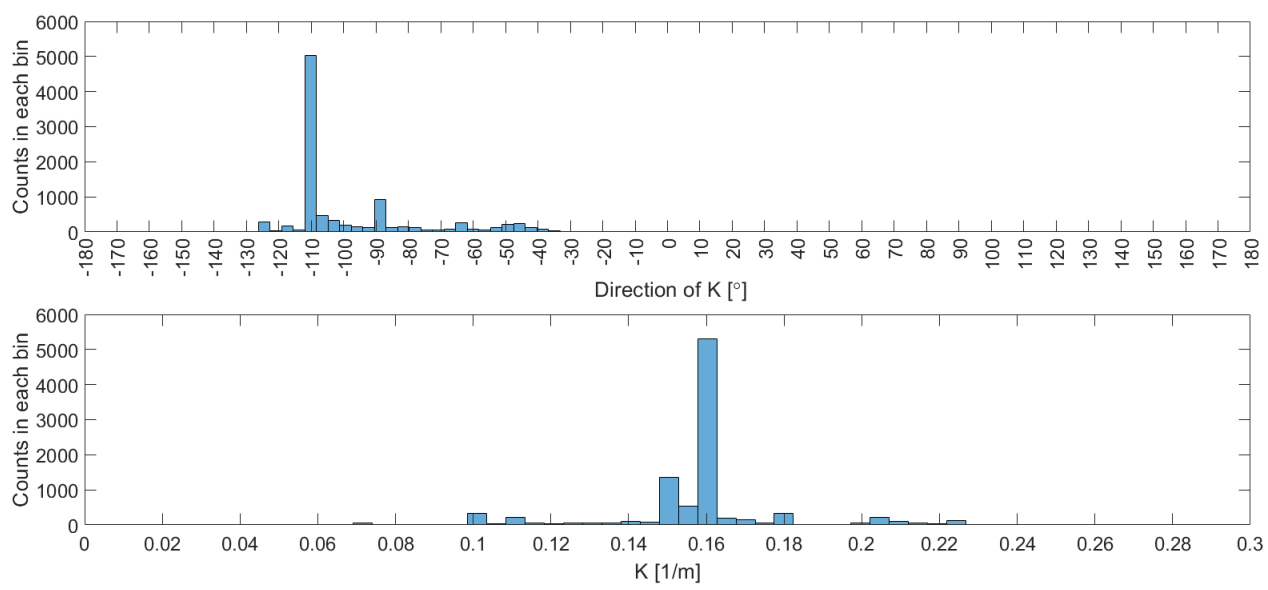

Figure 8. Histograms of direction (top) and module (bottom) of the wave-vector for frequency 17.1 Hz. Bin width: $3.6^{\circ}$ (top), $5 \times 10^{-3} 1 / \mathrm{m}$ (bottom).
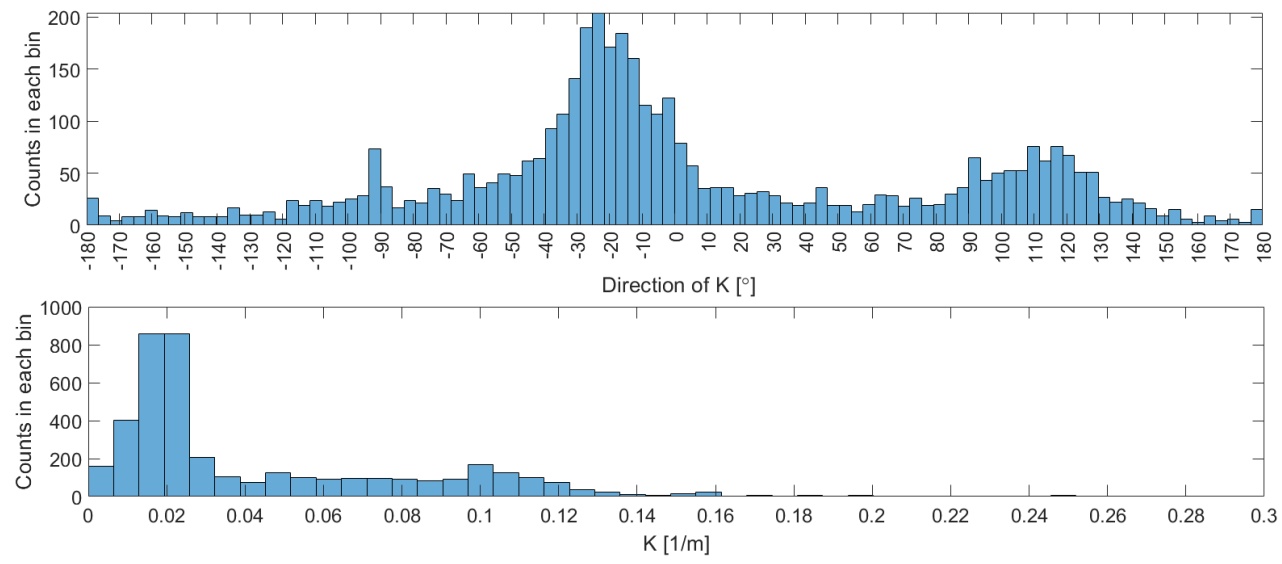

Figure 9. Histograms of direction (top) and module (bottom) of the wave-vector for frequency $5.5 \mathrm{~Hz}$. Bin width: $3.6^{\circ}$ (top), $5 \times 10^{-3} 1 / \mathrm{m}$ (bottom).

For frequency $17.1 \mathrm{~Hz}$ results indicate the direction $-110 \pm 2.5^{\circ}$ and wavenumber $\left|K_{17.1}\right|=0.1581 / \mathrm{m}$. Using this formula these values correspond to the seismic wave arriving from northeast with velocity $680 \pm 111 \mathrm{~m} / \mathrm{s}$. The $5.5 \mathrm{~Hz}$ wavefield direction is ca. $-25 \pm 16.3^{\circ}$ with wavenumber $\left|K_{17.1}\right|=0.021 / \mathrm{m}$. These values correspond to the seismic wave arriving from northwest with velocity $1727 \pm 431 \mathrm{~m} / \mathrm{s}$.

In Figure 10 the map of the vicinity of the Virgo detector with directions of waves propagation is shown. Red lines is an assumed coordination system, shifted $19.5^{\circ}$ clockwise to cardinal north. Blue lines indicate direction of $5.5 \mathrm{~Hz}$ wave with errors. The area in blue rectangle, i.e., an industrial complex $2.5 \mathrm{~km}$ away from the WEB, may be a source of this wave. Black color indicates direction of $17.1-\mathrm{Hz}$ wave. In the top-right corner of Figure 10 a zoom of the vicinity of the WEB is shown, with a potential source of $17.1 \mathrm{~Hz}$ wave marked (black rectangle), ca. $100 \mathrm{~m}$ away for the WEB. 


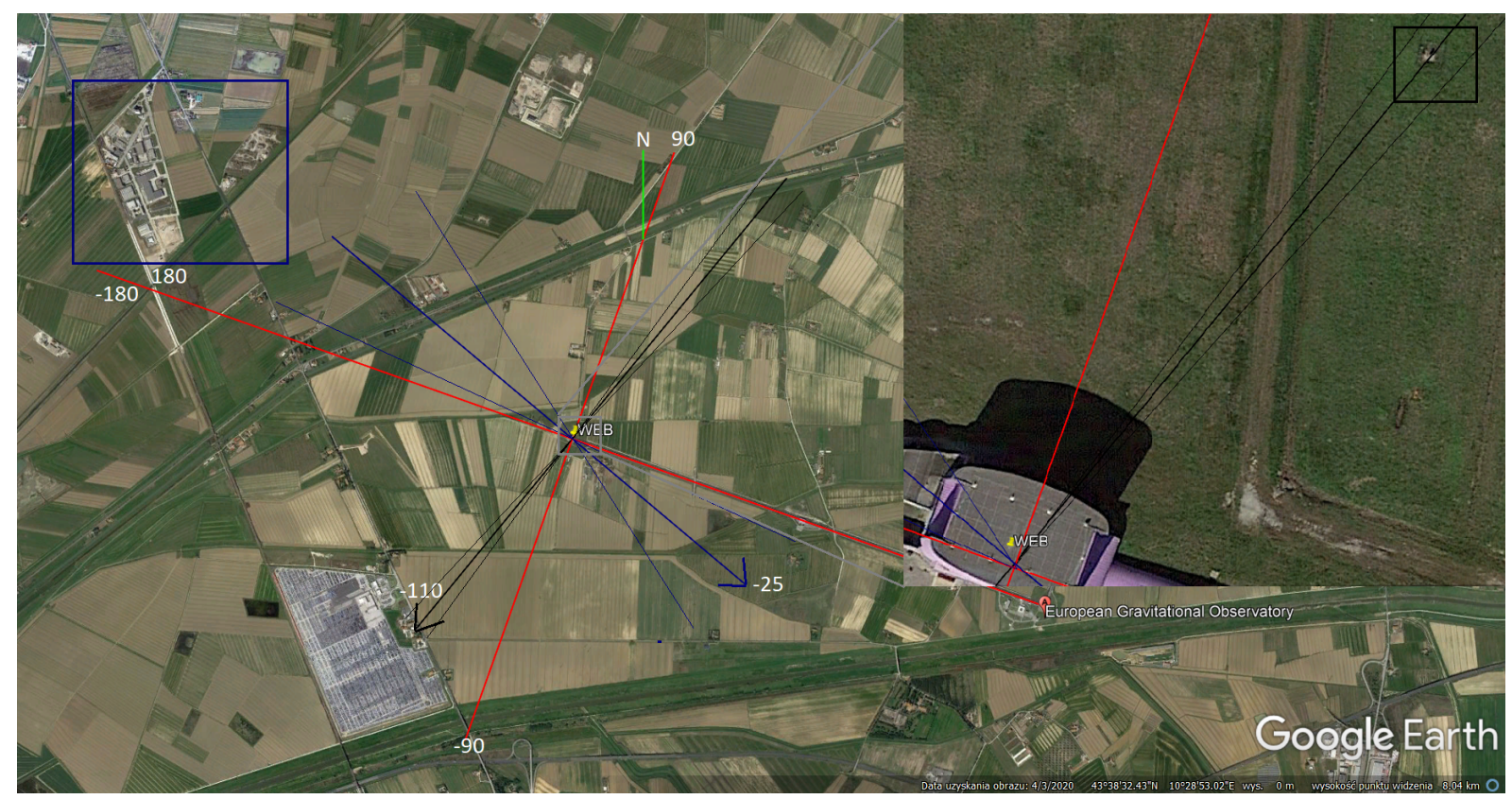

Figure 10. Map of the vicinity of the Virgo WEB $(8 \times 4 \mathrm{~km})$. Red lines indicate coordination system consistent with buildings. The directions of waves propagation for $5.5 \mathrm{~Hz}$ wave with error $\pm 16.3^{\circ}$ (blue arrow + side lines) and for $17.1 \mathrm{~Hz}$ wave with error $\pm 2.5^{\circ}$ (black arrow + side lines). Rectangles indicate the possible location of wave sources. The area close to the $17.1 \mathrm{~Hz}$ wave source is zoomed and shown in the top-right corner of the figure.

\section{Discussion}

Using the data from the experiment conducted between 24 January 2018 and 6 February 2018 collected by the system of seismometers inside the west-end building of Virgo, we made a seismic analysis of the nearby area.

We searched for the seismic waves with a frequency from 5 to $20 \mathrm{~Hz}$. Two dominant frequencies were detected and associated with possible sources, the first one is present most of the time, the second one appears primarily during daytime. First observed dominant frequency is $17.1 \mathrm{~Hz}$. The direction of this wave is $-110 \pm 2.5^{\circ}$, i.e., it was arriving from northeast, with velocity $680 \pm 111 \mathrm{~m} / \mathrm{s}$. In Figure 10 top-right corner a small object located ca. $100 \mathrm{~m}$ from WEB matches the found wave direction. It is located exactly at the found wave direction. However, this is a technical construction used for controlling the hydrology of the terrain and it is not a likely source of the $17.1-\mathrm{Hz}$ wave. For the most likely source of the $17.1-\mathrm{Hz}$ wave see [29].

Seismic waves at the frequency $5.5 \mathrm{~Hz}$ appear associated with daily human activity. They dominate between 6 a.m. and 6 p.m. every day and move with velocity $1727 \pm 431 \mathrm{~m} / \mathrm{s}$. A direction of this waves is not strict, ca. $-25 \pm 16.3^{\circ}$. A supposed origin of the waves is human activity and heavy traffic activity in the nearby industrial complex. Previous studies indicated another antropogenic source of the noise at the frequency 4-10 Hz: nearby high-traffic elevated roads [23,25]. The directional analysis we did is not fully consistent with such an interpretation.

The velocities given above are typical of dispersion media, i.e., the wave with lower frequency moves faster, and agree with the predictions of one of the models from [8]. The typical velocity of Rayleigh waves in metals is $2-5 \mathrm{~km} / \mathrm{s}$, while in soil $50-700 \mathrm{~m} / \mathrm{s}$ for shallow waves (under $100 \mathrm{~m}$ ) and $1.5-4 \mathrm{~km} / \mathrm{s}$ for depth over $1 \mathrm{~km} \mathrm{[36].}$

\section{Future Work}

The method of seismic-wave detection in the Virgo WEB presented above, can detect specific waves that pass through the building. The main advantage of the method is that it provides the full information about the waves that pass through the measuring system 
(frequency, velocity, direction, and amplitude) with small loss of the information from the seismometers, as it does not use data averaging.

The concept of minimization of Newtonian noise in the Virgo GW signal is based on monitoring sources of gravitational field perturbations. These perturbations are caused by local changes of density in the vicinity of the test masses. A commonly proposed method for the reduction of Newtonian noise is based on Wiener filters, which have been already applied for the reduction of other noises in GW detectors [37]. The parameters of seismic waves found in this work can be used to fit correlation models of the Wiener filter [9].

The analysis conducted herein can be applied to other Virgo experimental areas, where the other test masses are located. For the west-end building itself the data were collected for more than one year; in the north-end building and central building a system of sensors is going to be installed as well. Complementarily to seismometer arrays, infrasound microphone arrays are going to be used for a characterization of the sources of gravitational field fluctuations caused by pressure changes. In particular, a detailed analysis of the frequencies below $10 \mathrm{~Hz}$ would be important, as for $1.7 \mathrm{~Hz}$ a significant noise coming from the nearby wind farm is observed [24].

One of the problems that needs solving when we use the spatio-temporal spectral analysis method is how to distinguish between the real waves and the noise. For instance, some threshold values may be used for this purpose [38,39]. However, in this article we focused on the dominant frequencies observed in the signals. The sensitivity is limited by the computational noise due to the non-uniform grid and is likely to be limited to waves with the amplitude not larger than $10 \%$ of the dominant wave as seen from Figure 1, where the signal is ca. 10 times larger in amplitude than the noise. Moreover, the issue of spatial aliasing should be resolved, e.g., by applying the window function also for the spatial dimensions (cf. Section 3). On top of that, we may consider the use of overlapping time periods when calculating the spectra, to improve the accuracy and relevance of the results.

Author Contributions: Conceptualization, M.D. and T.B.; methodology, M.D. and T.B.; software, M.D. and R.S.; validation, R.S., M.D. and T.B.; formal analysis, R.S., M.D. and T.B.; investigation, R.S., M.D. and T.B.; resources, A.K., K.N., M.S., R.S., M.D. and T.B.; data curation, R.S., M.D. and T.B.; writing—original draft preparation, M.D.; writing—review and editing, R.S., T.B., M.D. and B.I.; visualization, R.S. and M.D.; supervision, T.B. and M.D.; project administration, T.B. and M.D.; funding acquisition, T.B. All authors have read and agreed to the published version of the manuscript.

Funding: This research was funded by Foundation for Polish Science grant numbers TEAM/20163/19 and MAB 3/2016 (AstroCeNT_Particle Astrophysics Science and Technology Centre International Research Agenda carried out within the International Research Agendas program of the Foundation for Polish Science financed by the European Union under the European Regional Development Fund).

Institutional Review Board Statement: Not applicable.

Informed Consent Statement: Not applicable.

Data Availability Statement: Not applicable.

Acknowledgments: M.D. is grateful for inspiring discussion with Krzysztof Lorek and Jan Harms. The authors are grateful to colleagues from the Virgo collaboration and the Virgo Newtonian noise team for providing the seismic array data and for fruitful discussions.

Conflicts of Interest: The authors declare no conflict of interest.

\author{
Abbreviations \\ The following abbreviations are used in this manuscript: \\ GW Gravitational wave \\ WEB West-end building (of Virgo) \\ ASD Amplitude spectral density \\ FFT, FT Fast Fourier transform \\ NUFFT Non-uniform fast Fourier transform
}




\section{Appendix A. Coordinates of Seismic Sensors}

Table A1. Coordinates of the seismometers with respect to the Virgo test mass.

\begin{tabular}{|c|c|c|c|}
\hline No. & $x[m]$ & $y[m]$ & $z[m]$ \\
\hline 1 & -2996.1855 & 3.3109 & -3.4011 \\
\hline 2 & -2998.6876 & 7.1706 & -3.4071 \\
\hline 3 & -2999.3596 & 3.2421 & -3.4008 \\
\hline 4 & -3003.9149 & 7.8757 & -3.4227 \\
\hline 5 & -3003.0811 & 3.2749 & -3.4150 \\
\hline 6 & -3005.9728 & 7.7190 & -3.4288 \\
\hline 7 & -3006.2434 & 3.3279 & -3.4237 \\
\hline 8 & -3010.3192 & 7.9888 & -3.4437 \\
\hline 9 & -3012.1375 & 3.3488 & -3.4299 \\
\hline 10 & -3014.7164 & 7.5541 & -3.4490 \\
\hline 11 & -3017.8701 & 4.2944 & -3.4613 \\
\hline 12 & -3014.9770 & -0.0874 & -3.4426 \\
\hline 13 & -3017.9104 & -4.0994 & -3.4595 \\
\hline 14 & -3015.1017 & -7.3702 & -3.4573 \\
\hline 15 & -3013.7171 & -3.1819 & -3.4287 \\
\hline 16 & -3011.1778 & -6.0311 & -3.4400 \\
\hline 17 & -3009.9611 & -3.1498 & -3.4302 \\
\hline 18 & -3005.5196 & -6.1313 & -3.4285 \\
\hline 19 & -3005.9568 & -3.1540 & -3.4234 \\
\hline 20 & -3003.6825 & -6.6868 & -3.4195 \\
\hline 21 & -3000.1694 & -3.4201 & -3.4149 \\
\hline 22 & -2999.4935 & -5.4172 & -3.4146 \\
\hline 23 & -2996.6250 & -3.3377 & -3.4085 \\
\hline 24 & -2999.3105 & 2.7366 & -3.3156 \\
\hline 25 & -3002.9757 & 2.6436 & -3.3247 \\
\hline 26 & -3006.8761 & 2.7944 & -3.3304 \\
\hline 27 & -3010.9353 & 2.8204 & -3.3416 \\
\hline 28 & -3013.8210 & 2.3477 & -3.3491 \\
\hline 29 & -3013.6882 & -2.6371 & -3.3435 \\
\hline 30 & -3011.2803 & -2.5983 & -3.3446 \\
\hline 31 & -3006.4946 & -2.6779 & -3.3386 \\
\hline 32 & -3002.6733 & -2.5689 & -3.3296 \\
\hline 33 & -2999.6753 & -2.5736 & -3.3223 \\
\hline 34 & -2999.8896 & 0.0321 & -3.3182 \\
\hline 35 & -3009.4183 & 0.0789 & -3.3497 \\
\hline 36 & -3013.7110 & -0.0613 & -3.3462 \\
\hline 37 & -3012.2423 & -1.1159 & -6.8445 \\
\hline 38 & -3008.4083 & 1.4434 & -6.8571 \\
\hline
\end{tabular}

\section{References}

1. Abbott, B.P.; Abbott, R.; Abbott, T.D.; Abernathy, M.R.; Acernese, F.; Ackley, K.; Adams, C.; Adams, T.; Addesso, P.; Adhikari, R.X.; et al. Observation of gravitational waves from a binary black hole merger. Phys. Rev. Lett. 2016, 116, 6. [CrossRef] [PubMed]

2. Sesana, A. Prospects for multiband gravitational-wave astronomy after GW150914. Phys. Rev. Lett. 2016, 116, 23. [CrossRef]

3. Endlich, S.; Penco, R. A modern approach to superradiance. J. High Energy Phys. 2017, 2017, 5. [CrossRef]

4. Sasaki, M.; Suyama, T.; Tanaka, T.; Yokoyama, S. Primordial black holes-perspectives in gravitational wave astronomy. Class. Quantum Gravity 2018, 35, 6. [CrossRef]

5. Miller, M.; Coleman, M.; Yunes, N. The new frontier of gravitational waves. Nature 2019, 568, 7753. [CrossRef] [PubMed]

6. Abbott, B.P.; Abbott, R.; Abbott, T.D.; Abraham, S.; Acernese, F.; Ackley, K.; Adams, C.; Adhikari, R.X.; Adya, V.B.; Affeldt, C.; et al. Tests of general relativity with the binary black hole signals from the LIGO-Virgo catalog GWTC-1. Phys. Rev. D 2019, 100, 10. [CrossRef]

7. Saulson, P.R. Terrestrial gravitational noise on a gravitational wave antenna. Phys. Rev. D 1984, 30, 4. [CrossRef] 
8. Hughes, S.A.; Thorne, K.S. Seismic gravity-gradient noise in interferometric gravitational-wave detectors. Phys. Rev. D 1998, 58, 12. [CrossRef]

9. Coughlin, M.; Mukund, N.; Harms, J.; Driggers, J.; Adhikari, R.; Mitra, S. Towards a first design of a Newtonian-noise cancellation system for Advanced LIGO. Class. Quantum Gravity 2016, 33, 24. [CrossRef]

10. Fiori, I.; Paoletti, F.; Tringali, M.C.; Janssens, K.; Karathanasis, C.; Menendez-Vazquez, A.; Romero-Rodriguez, A.; Sugimoto, R.; Washimi, T.; Boschi, V.; et al. The hunt for environmental noise in virgo during the third observing run. Galaxies $2020,8,82$. [CrossRef]

11. Acernese, F.; Agathos, M.; Agatsuma, K.; Aisa, D.; Allemandou, N.; Allocca, A.; Amarni, J.; Astone, P.; Balestri, G.; Ballardin, G.; et al. Advanced Virgo: A second-generation interferometric gravitational wave detector. Class. Quantum Gravity $2014,32,2$. [CrossRef]

12. Hartmann, D.L. ATM S 552 Notes. pp. 188-191. Available online: https://atmos.washington.edu/ dennis/552_Notes_ftp.html (accessed on 13 July 2021)

13. Lacoss, R.T.; Kelly, E.J.; Toksöz, M.N. Estimation of seismic noise structure using arrays. Geophysics 1969, 34, 1. [CrossRef]

14. Gazdag, J.; Sguazzero, P. Migration of seismic data. Proc. IEEE 1984, 72, 10. [CrossRef]

15. Mainsant, G.; Larose, E.; Broennimann, C.; Jongmans, D.; Michoud, C.; Jaboyedoff, M. Ambient seismic noise monitoring of a clay landslide: Toward failure prediction. J. Geophys. Res. Earth Surf. 2012, 117, F1. [CrossRef]

16. Kao, S.K. Governing equations and spectra for atmospheric motion and transports in frequency, wave-number space. J. Atmos. Sci. 1968, 25, 1. [CrossRef]

17. Hayashi, Y. Space-time spectral analysis and its applications to atmospheric waves. J. Meteorol. Soc. Jpn. Ser. II 1982, 60, 1. [CrossRef]

18. Salby, M.L. Sampling theory for asynoptic satellite observations. Part I: Space-time spectra, resolution, and aliasing. J. Atmos. Sci. 1982, 39, 11. [CrossRef]

19. Wu, D.L.; Hays, P.B.; Skinner, W.R. A least squares method for spectral analysis of space-time series. J. Atmos. Sci. 1995, 52, 20. [CrossRef]

20. Hendon, H.H.; Wheeler, M.C. Some space-time spectral analyses of tropical convection and planetary-scale waves. J. Atmos. Sci. 2008, 65, 9. [CrossRef]

21. De Michele, C.; Bernardara, P. Spectral analysis and modeling of space-time rainfall fields. Atmos. Res. 2005, 77, 1-4. [CrossRef]

22. Kim, G.; Barros, A.P. Space-time characterization of soil moisture from passive microwave remotely sensed imagery and ancillary data. Remote Sens. Environ. 2002, 81, 2-3. [CrossRef]

23. Acernese, F.; Amico, P.; Arnaud, N.; Babusci, D.; Barille, R.; Barone, F.; Barsotti, L.; Barsuglia, M.; Beauville, F.; Bizouard, M.A.; et al. Properties of seismic noise at the Virgo site. Class. Quantum Gravity 2004, 21, 5. [CrossRef]

24. Saccorotti, G.; Piccinini, D.; Cauchie, L.; Fiori, I. Seismic noise by wind farms: A case study from the Virgo Gravitational Wave Observatory, Italy. Bull. Seismol. Soc. Am. 2011, 101, 2. [CrossRef]

25. Koley, S.; Bulten, H.J.; van den Brand, J.; Bader, M.; Campman, X.; Beker, M. S-wave velocity model estimation using ambient seismic noise at Virgo, Italy. In SEG Technical Program Expanded Abstracts 2017; Society of Exploration Geophysicists: Tulsa, OK, USA, 2017; pp. 2946-2950.

26. Somlai, L.A.; Graczer, Z.; Levai, P.; Vasuth, M.; Weber, Z.; Van, P. Seismic noise measures for underground gravitational wave detectors. Acta Geod. Geophys. 2019, 54, 2. [CrossRef]

27. Ván, P.; Barnafoldi, G.G.; Bulik, T.; Biro, T.; Czellar, S.; Cieslar, M.; Czanik, C.; David, E.; Debreceni, E.; Denys, M.; et al. Long term measurements from the Mátra Gravitational and Geophysical Laboratory Eur. Phys. J. 2019, 228, 8.

28. Mukund, N.; Coughlin, M.; Harms, J.; Biscans, S.; Warner, J.; Pele, A.; Thorne, K.; Barker, D.; Arnaud, N.; Donovan, F.; et al. Ground motion prediction at gravitational wave observatories using archival seismic data. Class. Quantum Gravity 2019, 36, 8. [CrossRef]

29. Tringali, M.C.; Bulik, T.; Harms, J.; Fiori, I.; Paoletti, F.; Singh, N.; Idzkowski, B.; Kutynia, A.; Nikliborc, K.; Suchiński, M.; et al. Seismic array measurements at Virgo's west end building for the configuration of a Newtonian-noise cancellation system. Class. Quantum Gravity 2019, 37, 2. [CrossRef]

30. Seismic Sensor Specification. Available online: http://www.innoseis.com/tremornet/ (accessed on 13 July 2021).

31. Beker, M.; Campman, X.; van Oven, J.; Walk, W.; Levell, J.; Tang, Z.; Danilouchkine, M.; Smit, D.; van den Brand, J.; Koley, S.; et al. Innovations in seismic sensors driven by the search for gravitational waves. Lead. Edge 2016, 35, 7. [CrossRef]

32. Singha, A.; Hild, S.; Harms, J. Newtonian-noise reassessment for the Virgo gravitational-wave observatory including local recess structures. Class. Quantum Gravity 2020, 37, 10. [CrossRef]

33. Dutt, A.; Rokhlin, V. Fast Fourier transforms for nonequispaced data. SIAM J. Sci. Comput. 1993, 14, 6. [CrossRef]

34. Greengard, L.; Lee, J.Y. Accelerating the nonuniform fast Fourier transform. SIAM Rev. 2004, 46, 3. [CrossRef]

35. Lee, Y.L.; Greengard, L. The type 3 nonuniform FFT and its applications. J. Comput. Phys. 2005, 206, 1. [CrossRef]

36. Telford, M.W.; Geldart, L.P.; Sheriff, R.E. Applied Geophysics; Cambridge University Press: New York, NY, USA, $1990 ;$ p. 149. 
37. Cuoco, E.; Cella, G.; Guidi, G.M. Whitening of non-stationary noise from gravitational wave detectors. Class. Quantum Gravity 2004, 21, 5. [CrossRef]

38. Denys, M.; Gubiec, T.; Kutner, R.; Jagielski, M.; Stanley, H.E. Universality of market superstatistics. Phys. Rev. E 2016, $94,4$. [CrossRef]

39. Schäfer, J. A Space-Time Analysis of Tropospheric Planetary Waves in the Northern Hemisphere. J. Atmos. Sci. 1979, 36, 6. 